\title{
Nucleolar localization of Small G protein RhoA is associated with active RNA synthesis in human carcinoma HEp-2 cells
}

\author{
YUEYING LI ${ }^{*}$, YONG HU ${ }^{2 *}$, LILONG CHE ${ }^{1}$, JUNHAI JIA ${ }^{1}$ and MIN CHEN ${ }^{1}$ \\ ${ }^{1}$ Department of Physiology, School of Medicine, Jiangsu University, Zhenjiang, Jiangsu 212013; ${ }^{2}$ Department of Neonatology, \\ Shanghai Children's Hospital, Shanghai Jiao Tong University, Shanghai 200040, P.R. China
}

Received May 16, 2015; Accepted March 1, 2016

DOI: $10.3892 / \mathrm{ol} .2016 .4450$

\begin{abstract}
Previous studies have demonstrated that the nuclear localization of ras homolog family member A (RhoA), with prominent concentration in the nucleolus, is a common feature in human cancer tissues and cancer cell lines. Although a previous study has demonstrated that the nuclear translocation of RhoA occurs via active transport, a process that occurs through importin $\alpha$ in a nuclear factor- $\kappa \mathrm{B}$-dependent manner, the mechanism, biological function and pathological meaning of the nucleolar residency of RhoA remain to be elucidated. As the cell nucleolus is the site of ribosome biosynthesis, the aim of the present study was to investigate the association between RNA synthesis and the nucleolar localization of RhoA, as well as the molecular mechanisms underlying the residency of RhoA in the nucleolus of HEp-2 (human larynx epithelial carcinoma) cells. Indirect immunofluorescence microscopy was used to evaluate the subcellular distribution of nuclear RhoA, and immunoblotting analysis was used to determine the total cellular protein level of RhoA. Consistent with the results of previous studies, untreated HEp-2 cells exhibited bright nucleolar staining, indicating an increased concentration of RhoA in the nucleoli. Treatment with actinomycin D for the inhibition of RNA synthesis caused a redistribution of RhoA from the nucleoli to the nucleoplasm with a speckled staining pattern. Immunoblotting revealed that neither the total cellular amount of RhoA nor the integrity of RhoA was affected by treatment with actinomycin $\mathrm{D}$. In cells that were treated at a decreased concentration $(0.05 \mathrm{mg} / \mathrm{l})$ of actinomycin $\mathrm{D}$, the redistribution of RhoA was reversible following the removal of the drug from the culture medium. However, this reversal was not observed at an increased drug concentration $(1 \mathrm{mg} / \mathrm{l})$.
\end{abstract}

Correspondence to: Professor Min Chen, Department of Physiology, School of Medicine, Jiangsu University, 301 Xuefu Road, Zhenjiang, Jiangsu 212013, P.R. China

E-mail: chenmina88@yahoo.com

*Contributed equally

Key words: ras homolog family member A, cell nucleus, RNA synthesis, actinomycin D, human cancer cells
Overall, to the best of our knowledge, the results of the present study provide the first in situ evidence that the inhibition of RNA synthesis induces a redistribution of nucleolar RhoA to the nucleoplasm, and additionally suggest that the nucleolar residency of RhoA in HEp-2 cells may be associated with active RNA synthesis.

\section{Introduction}

The cell nucleolus is a dynamic, non-membrane-bound subnuclear structure, which has a classically well-established role in ribosome biogenesis, including the synthesis and processing of the ribosomal RNA (rRNA) precursor molecules (pre-rRNAs) and the assembly of pre-rRNAs with particular ribosomal and nonribosomal proteins to form preribosomal particles $(1,2)$. Mammalian nuclei typically contain one nucleolus, whereas tumor cells tend to contain several nucleoli (3). The typical tripartite organization of the nucleolus in mammals, which accounts for ribosomal gene transcription and pre-rRNA processing efficiency, is composed of three distinct components: The fibrillar center (FC), the dense fibrillar component (DFC) and the granular component (GC) (4-7). The early accumulation and processing of rRNA takes place in the DFC, whereas late rRNA processing and building of the ribosomal components occurs in the GC (2). As well as small nucleolar RNAs, a number of proteins, including fibrillarin and nucleolar phosphoprotein B23 (B23), are located in the nucleolus (6). With regard to the unique nulceolar localization in the cells at interphase, fibrillarin and B23 are also widely used as markers for the DFC and GC, respectively (8).

Previous studies have indicated that the nucleolus is plurifunctional $(9,10)$. In addition to the typically recognized function of ribosome biosynthesis, the nucleolus supports the production of additional gene expression components, including transfer RNA and translational apparatus, the signal recognition particle, ribonucleoprotein enzyme involved in maintenance of telomerase integrity and U6 RNA, a catalytic RNA of the spliceosome (9).

Small G protein ras homolog family member A (RhoA), with a molecular weight of $21 \mathrm{kDa}$, is the most extensively studied member of the Rho guanosine triphosphate (GTP)ase family and is part of the Ras super family of small $\mathrm{G}$ proteins (11). RhoA has been reported to regulate numerous biological activities, including gene transcription (12) and 
tumor progression (13). Recent research on the intracellular localization of RhoA has shown that it is located not only in the cytosol and cell membrane, but also in the cell nucleus (14-16). Notably, previous studies have demonstrated that the nuclear localization of RhoA with predominant concentration in the cell nucleolus is a common feature in human cancer tissues and cell lines $(17,18)$. Although our previous study has demonstrated that the nuclear translocation of RhoA is via active transport, a process involving importin $\alpha$ in a nuclear factor- $\kappa \mathrm{B} 1$-dependent manner, the mechanism, biological function and pathological meaning of the nucleolar residency of RhoA remain to be elucidated (18).

The antitumor antibiotic actinomycin D is a polypeptide antibiotic that is widely used as inhibitor of RNA synthesis. Actinomycin D is able to bind to duplex DNA and inhibit the progression of DNA-dependent RNA polymerase $(19,20)$. Studies on the subcellular distribution of B23 have demonstrated consistently that it is sensitive to actinomycin D treatment and translocates from the cell nucleoli to alternative regions of the nucleus (21-24). By contrast, the results of studies regarding the subcellular distribution of fibrillarin with actinomycin D treatment are diverse (25-27). A previous study demonstrated that nucleolar fibrillarin also redistributed to nucleoplasmic entities in response to actinomycin D treatment in HEp-2 cells (28).

By utilizing an RNA synthesis inhibitor, the present study aimed to investigate the association between RNA synthesis and the nucleolar concentration of RhoA in human HEp-2 cells, as well as the potential molecular mechanisms underlying the nucleolar residency of RhoA.

\section{Materials and methods}

Cell lines and cell culture. Human carcinoma HEp-2 cells, which were originally derived from an epidermoid carcinoma of the larynx, purchased from the American Type Culture Collection (Manassas, VA, USA) were maintained in complete RPMI-1640 that contained 10\% fetal bovine serum (FBS) and was supplemented with L-glutamine ( $2 \mathrm{mmol} / \mathrm{l})$, salt pyruvate ( $1 \mathrm{mmol} / \mathrm{l}), 1 \%$ non-essential amino acids and streptomycin $(10 \mathrm{mg} / \mathrm{l})$, at $37^{\circ} \mathrm{C}$ in a humidified atmosphere of $5 \% \mathrm{CO}_{2}$. The cell culture medium was replaced every two days and the cells were maintained at subconfluence.

Reagents. RPMI-1640 culture medium and FBS were obtained from Gibco (Thermo Fisher Scientific, Inc., Waltham, MA, USA). L-glutamine, salt pyruvate, non-essential amino acids, streptomycin, phosphate-buffered saline (PBS) and nuclear fluorochrome Hoechst 33342 were obtained from Sigma-Aldrich (St. Louis, MO, USA). Primary antibodies against RhoA (monoclonal mouse; \#sc-418; dilution, 1:200) and fibrillarin (mouse monoclonal; \#sc-166000; dilution, 1:100) were obtained from Santa Cruz Biotechnology, Inc. (Dallas, TX, USA), whereas monoclonal mouse antibody against B23 (\#B0556; dilution, 1:100) was purchased from Sigma-Aldrich. Actinomycin D was additionally obtained from Sigma-Aldrich. Mouse monoclonal antibody against glyceraldehyde-3-phosphate dehydrogenase (GAPDH; \#KC-5G4; dilution, 1:5,000) was obtained from KangCheng Bio-tech Inc. (Shanghai, China). Goat anti-mouse immuno- globulin (Ig)G fluorescein isothiocyanate-conjugated (FITC; \#115-097-003; dilution, 1:1,000) and goat anti-mouse IgG horseradish peroxidase (HRP)-conjugated (\#115-035-209; dilution, 1:5,000) secondary antibodies were obtained from Jackson ImmunoResearch Laboratories, Inc. (West Grove, PA, USA). Enhanced chemiluminescence (ECL) reagents were purchased from GE Healthcare Life Sciences (Chalfont, UK).

Actinomycin D treatment. A stock solution of actinomycin D ( $2.5 \mathrm{~g} / \mathrm{l})$ was prepared in ethanol (Changzhou Yabang Chemical Co.,Ltd., Changzhou, China). It was diluted at least 2,500-fold and freshly prepared prior to addition to the cells. For the reversibility assay, HEp-2 cells grown on cover slips in a 6-well plate (Genetimes Biological Mall, Shanghai, China) were treated with actinomycin D. Following treatment, cover slips were washed 3 times (1 min each wash) in prewarmed PBS, followed by 1 brief wash with prewarmed fresh cell culture medium without actinomycin D. Subsequent to treatment with actinomycin D at the concentrations of $0.05 \mathrm{mg} / \mathrm{l}$ (for $4 \mathrm{~h}$ ) and $1 \mathrm{mg} / \mathrm{l}$ (for $1 \mathrm{~h}$ ), cells were cultivated in fresh culture medium without actinomycin D for an additional incubation of 24 or $27 \mathrm{~h}$, which was dependent on the re-accumulation of RhoA in the cell nucleolus.

Immunofluorescence microscopy. HEp-2 cells grown on cover slips were fixed with freshly prepared paraformaldehyde (Nantong Jiangtian Chemical Co., Ltd., Nangtong, China) (40 g/l in PBS) for $30 \mathrm{~min}$. Subsequent to being penetrated with $30 \mathrm{ml} / 1$ Triton X-100 (Beijing Solarbio Science \& Technology Co., Ltd., Beijing, China) for $8 \mathrm{~min}$ and blocked with $30 \mathrm{~g} / \mathrm{l}$ bovine serum albumin (Genetimes Biological Mall) for $1 \mathrm{~h}$, the cells were incubated with the primary antibodies against RhoA, fibrillarin or B23 at $4^{\circ} \mathrm{C}$ overnight, followed by another incubation with the species-specific anti-IgG FITC-conjugated secondary antibody for $1 \mathrm{~h}$ at room temperature. Cells were washed three times with PBS (10 min each wash) subsequent to each incubation. The distribution of the target protein in the cells was analyzed by confocal laser scanning microscopy (LSM 510; Carl Zeiss Microscopy GmbH, Jena, Germany). For the counterstaining of cell nuclei with B23 or fibrillarin, cells were incubated with nuclear fluorochrome Hoechst $33342(10 \mathrm{mg} / \mathrm{l})$ for $15 \mathrm{~min}$ at room temperature, followed by three washes in PBS.

Western blotting assay. Cells were washed with cold PBS and transferred to a $1.7 \mathrm{ml}$ centrifuge tube (Genetimes Biological Mall) where they were centrifuged at $200 \mathrm{x} \mathrm{g}$ for $5 \mathrm{~min}$ at $4^{\circ} \mathrm{C}$. Lysis buffer $(50 \mathrm{mM}$ Tris, $150 \mathrm{mM} \mathrm{NaCl}, 1 \mathrm{mM}$ ethylenediaminetetraacetic acid and $1 \%$ Triton $\mathrm{X}-100 ; \mathrm{pH}, 7.4$; Sinopharm Chemical Reagent Co., Ltd., Shanghai, China) was added according to the proportion of $1 \mathrm{ml}$ lysis buffer in $10^{7}$ cells. Following agitation for $15 \mathrm{~min}$ at $4^{\circ} \mathrm{C}$, centrifugation at $12,000 \times \mathrm{g}$ was performed for $15 \mathrm{~min}$ at $4^{\circ} \mathrm{C}$. The supernatant was collected as whole cell protein extract. The sample proteins of whole HEp- 2 cell lysate (20 $\mu \mathrm{g}$ protein) were run on $12.5 \%$ sodium dodecyl sulfate-polyacrylamide gels, followed by transferal onto polyvinyl difluoride (PVDF) membranes (Bio-Rad Laboratories, Inc., Hercules, CA, USA). GAPDH was used as a loading control. The PVDF membranes were initially blocked with $5 \%$ milk in TBS-T $(\mathrm{NaCl}, 80 \mathrm{~g} / \mathrm{l}$; 

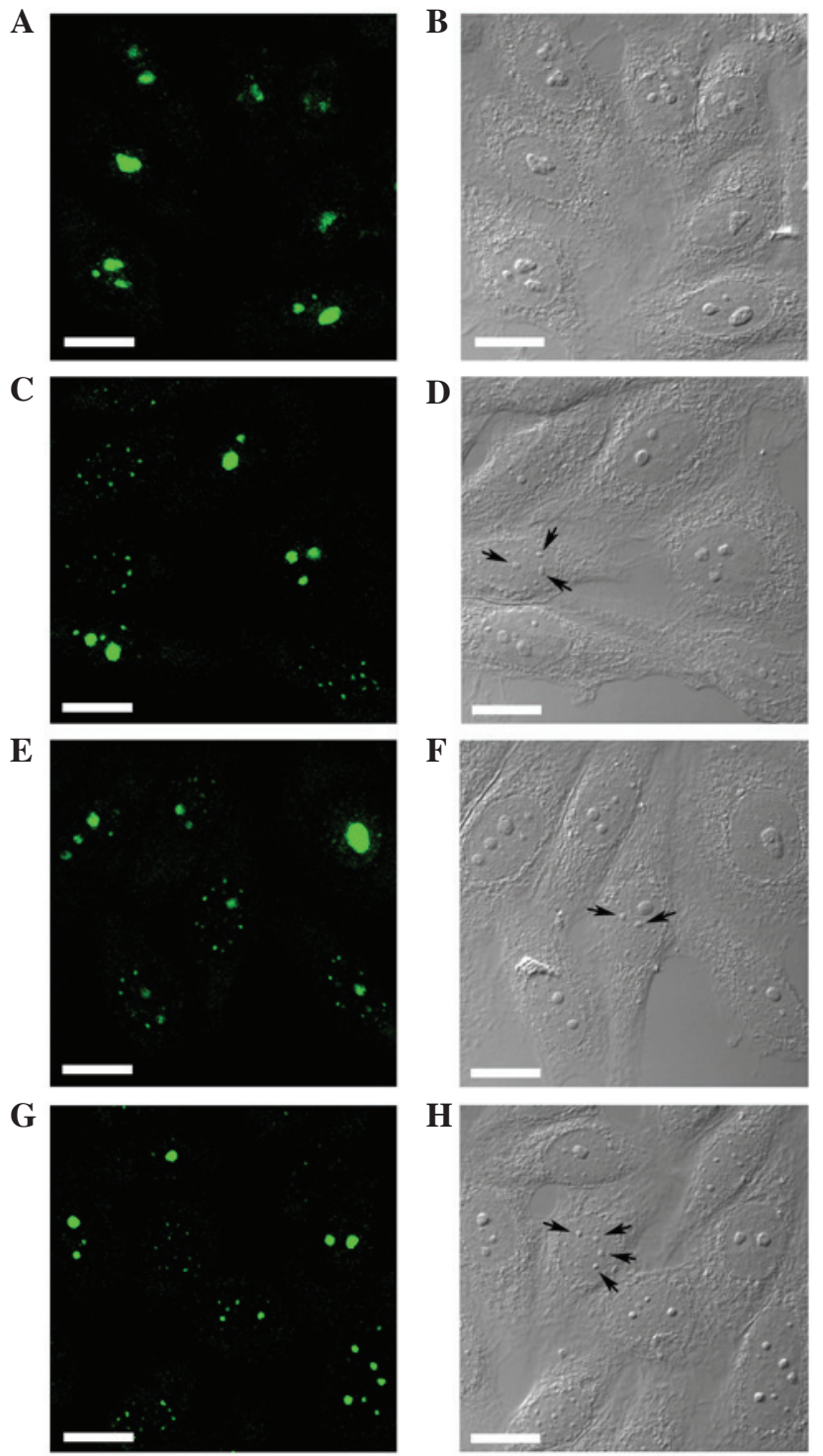

Figure 1. Alteration of the intracellular localization of RhoA induced by actinomycin D in HEp-2 cells. (A) Indirect immunofluorescence revealed prominent nucleolar RhoA in untreated cells. (B) Corresponding cells were revealed in differential interference contrast pictures. Redistribution of nucleolar RhoA to nucleoplasmic speckles was observed in the cells treated with actinomycin D at the concentrations of (C) $0.05 \mathrm{mg} / 1$, (D) differential interference contrast; (E) $0.10 \mathrm{mg} / \mathrm{l}$, (F) differential interference contrast; and $(\mathrm{G}) 1.00 \mathrm{mg} / 1$ for $4 \mathrm{~h},(\mathrm{H})$ differential interference contrast. Arrows represent the segregated nucleoli or nucleolus-like bodies. Scale bar, $10 \mu \mathrm{m}$. RhoA, ras homolog family member A.

KCl, 2 g/1; Tris, 30 g/l; Tween-20, 0.1\%; pH 7.4; Sinopharm Chemical Reagent Co., Ltd.) for $1 \mathrm{~h}$ at room temperature and subsequently incubated with the primary antibodies against RhoA and GAPDH at $4^{\circ} \mathrm{C}$ overnight. Following the incubation of the membranes with the anti-IgG HRP-conjugated secondary antibody for $1 \mathrm{~h}$ at room temperature, ECL reagents were used to indicate the positive bands on the membrane, according to the manufacturer's protocol. The bands were detected using the Typhoon 9400 (GE Healthcare Life Sciences).

Statistical analysis. A comparison of the amount of total RhoA protein following treatment with varying concentrations of actinomycin D was evaluated by one-way analysis of variance using SPSS 16.0 software (SPSS, Inc., Chicago, IL, USA).
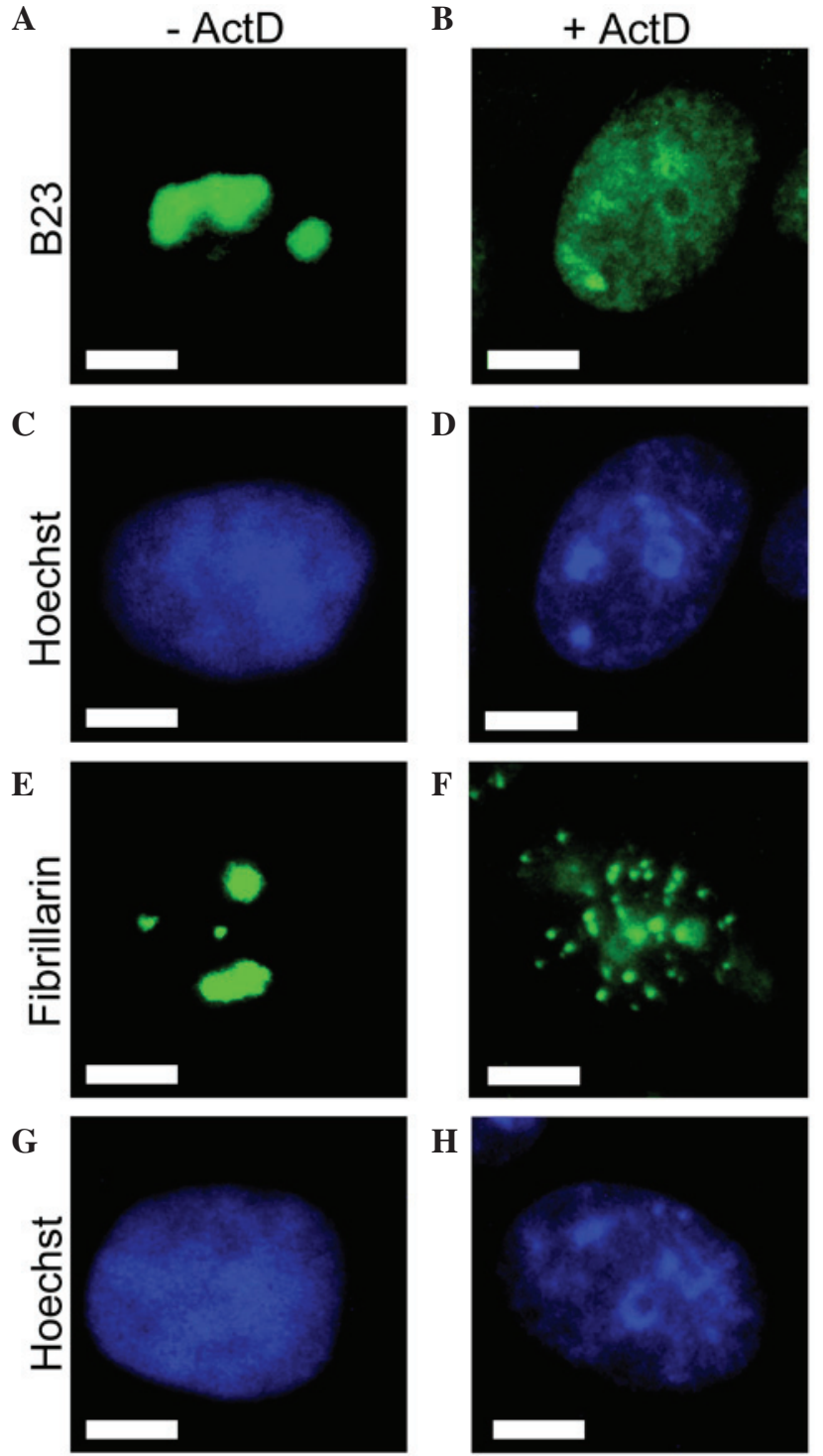

Figure 2. Actinomycin D altered the subcellular location of nucleolar proteins B23 and fibrillarin in HEp-2 cells. Cells showed nucleolar localization of B23 (A) without treatment and (B) with $0.05 \mathrm{mg} / 1$ actinomycin D for $4 \mathrm{~h}$. B23 translocated from the nucleoli to the nucleoplasm in a diffuse staining pattern. (C and D) Corresponding cell nuclei were shown by nuclear staining with Hoechst 33342. Cells demonstrated nucleolar localization of fibrillarin (E) without treatment and (F) with $0.05 \mathrm{mg} / \mathrm{l}$ actinomycin D for $4 \mathrm{~h}$. Fibrillarin redistributed to small nucleoplasmic entities. ( $\mathrm{G}$ and $\mathrm{H}$ ) Corresponding cell nuclei were shown by nuclear staining with Hoechst 33342. Scale bar, $5 \mu \mathrm{m}$. B23, nucleolar phosphoprotein B23.

$\mathrm{P}<0.05$ was considered to indicate a statistically significant difference.

\section{Results}

The subcellular redistribution of nucleolar RhoA following actinomycin D treatment in HEp-2 cells. Confocal laser scanning microscopy revealed the nuclear localization of RhoA with predominant accumulation in the cell nucleolus in HEp-2 cells with no drug treatment (Fig. 1A), whereas treatment with actinomycin D altered the subcellular distribution of RhoA within the cell nucleus. Corresponding differential interference contrast pictures revealed large nucleoli in untreated HEp-2 cells (Fig. 1B). Accompanied by a decrease in nucleolar 


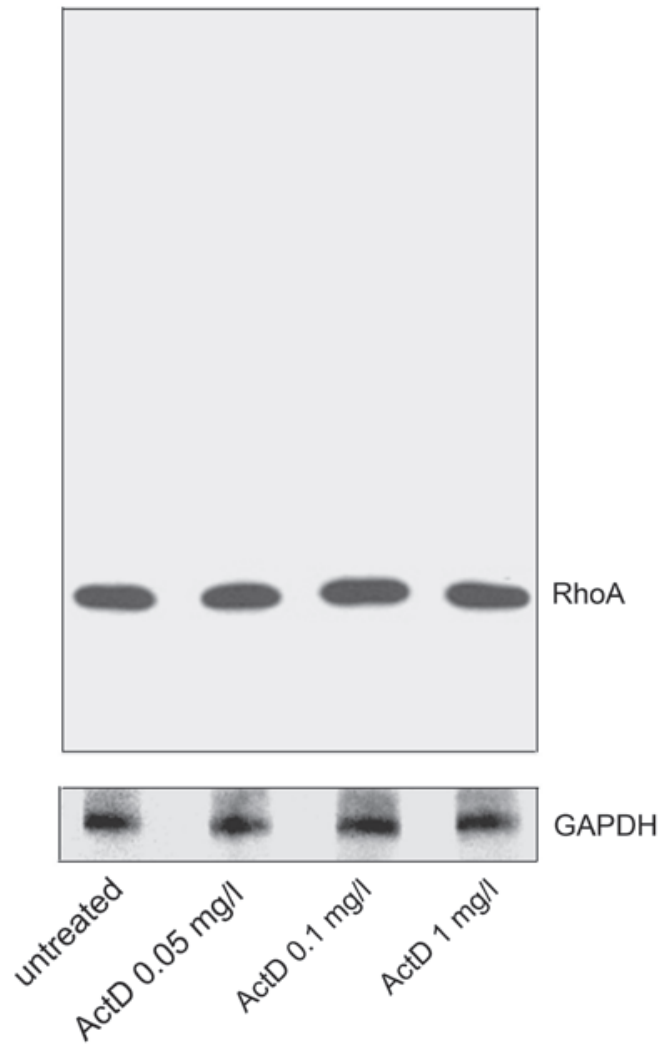

Figure 3. Immunoblotting of RhoA protein amount in whole HEp-2 cell lysate Total RhoA protein was detected as a single band at $21 \mathrm{kDa}$ in untreated cells and cells treated with actinomycin D for $4 \mathrm{~h}$ at the concentration of $0.05 \mathrm{mg} / 1$, $0.1 \mathrm{mg} / \mathrm{l}$ or $1 \mathrm{mg} / \mathrm{l}$. Glyceraldehyde-3-phosphate dehydrogense was used as a loading control. RhoA, ras homolog family member A.

staining, a bright nucleoplasmic speckle staining of RhoA was visualized in the cells (Fig. 1C, E and G). However, the segregated nucleoli or nucleolus-like bodies were observed in the cells treated with actinomycin D (Fig. 1D, F and 1H; arrows).

Altered subcellular localization of nucleolar protein B23 in HEp-2 cells following actinomycin D treatment. Intracellular localization of two abundant nucleolar proteins fibrillarin and B23 was additionally examined by indirect immunofluorescence (Fig. 2). Nucleolar protein B23 is well-known to relocalize following treatment with actinomycin $\mathrm{D}$ in numerous cell types, including HEp-2 cells (21-28). The staining for B23 was concentrated in the nucleoli of untreated HEp-2 cells (Fig. 2A), but was redistributed diffusely throughout the nucleus following $4 \mathrm{~h}$ of treatment with $0.05 \mathrm{mg} / \mathrm{l}$ actinomycin D (Fig. 2B). This redistribution of B23 may act as a marker for effective treatment by actinomycin D. Another abundant nucleolar protein, fibrillarin, demonstrated exclusively nucleolar localization in untreated cells (Fig. 2E). Consistent with the previous finding, a redistribution of fibrillarin to the nucleoplasmic small entities was induced by actinomycin D (Fig. 2F).

RhoA protein content is unchanged following actinomycin D treatment. Following treatment with actinomycin D for $4 \mathrm{~h}$, HEp-2 cells were washed with PBS and lysed under denaturing conditions. The protein amounts from whole-cell lysate were compared through immunoblotting analysis. Regardless of
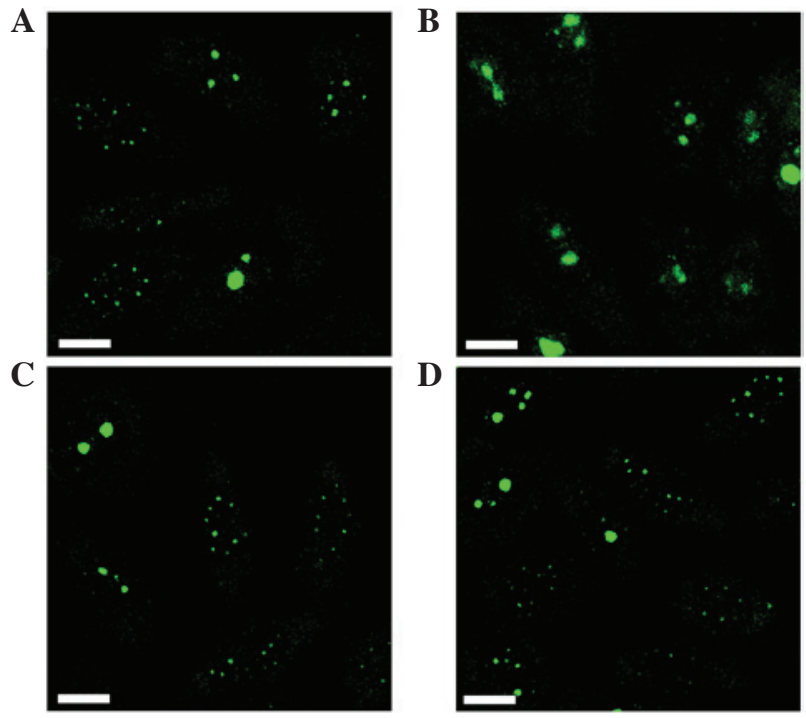

Figure 4. Localization of ras homolog family member A in HEp-2 cells following the removal of actinomycin D. (A) Cells treated with actinomycin D at a concentration of $0.05 \mathrm{mg} / 1$ for $4 \mathrm{~h}$. (B) Cells treated with actinomycin D at a concentration of $0.05 \mathrm{mg} / 1$ for $4 \mathrm{~h}$, washed and incubated for $24 \mathrm{~h}$ in drug-free culture medium. (C) Cells treated with actinomycin D at a concentration of $1 \mathrm{mg} / 1$ for $1 \mathrm{~h}$. (D) Cells treated with actinomycin D at a concentration of $1 \mathrm{mg} / 1$ for $1 \mathrm{~h}$, washed and incubated for $27 \mathrm{~h}$ in drug-free culture medium. Scale bar, $10 \mu \mathrm{m}$.

the various concentrations of actinomycin D, immunoblotting revealed a single band exclusively at $21 \mathrm{kDa}$ for all untreated and actinomycin D-treated groups (Fig. 3). Statistical analysis on the amount of total RhoA protein following various treatments with actinomycin D demonstrated no change in total cellular RhoA content $(\mathrm{P}>0.05)$. Therefore, the actinomycin $\mathrm{D}$ treatment did not affect the net protein amount of RhoA, whilst it did affect the cellular distribution of the RhoA protein.

Nuclear redistribution of RhoA is reversible. To investigate the persistence and reversibility of the actinomycin D-induced nuclear redistribution of RhoA, HEp-2 cells were initially treated with actinomycin $\mathrm{D}$, followed by an additional cultivation in fresh culture medium without actinomycin $\mathrm{D}$ for at least $24 \mathrm{~h}$. When HEp-2 cells were incubated with $0.05 \mathrm{mg} / \mathrm{l}$ actinomycin D for $4 \mathrm{~h}$, a redistribution of RhoA was observed (Fig. 4A). Removal of the drug from the culture medium allowed the reaccumulation of RhoA in the cell nucleoli $24 \mathrm{~h}$ later (Fig. 4B). However, reaccumulation was not observed following initial treatment with an increased concentration of actinomycin D (1 mg/l for $1 \mathrm{~h}$ ) (Fig. 4C), even when the incubation time without actinomycin D was extended to $27 \mathrm{~h}$ (Fig. 4D).

\section{Discussion}

Over the past few decades, the understanding of nucleolar function has changed markedly with the concept of the plurifunctional nucleolus (9). Proteomic studies have suggested that the cell nucleolus may be involved in a wide range of cellular processes independent of ribosome biogenesis (29). Extensive evidence indicates that the nucleolus affects the response to cellular stress, and regulates the cell cycle and cell growth (29). 
Perturbations to the nucleolus have been reported in a wide range of cellular diseases, from autoimmunity to cancer (30).

An important aspect regarding nucleolus structure and function are the morphological variations that exist between normal somatic and neoplastic or malignant cells (31). For over a century, an increase in the size and number of cell nucleoli has been utilized as a marker of aggressive tumors $(31,32)$. Contemporary studies have inferred that nucleoli may have a broad role in malignant transformation. Specifically, the extra-ribosomal functions of the nucleolus position the organelle as a central integrator of cellular proliferation and stress signaling and are emerging as important mechanisms for modulating how oncogenes and tumor suppressors operate in normal and malignant cells (33).

Research has demonstrated that RhoA serves a key role in governing extra- and intracellular signaling transduction, in addition to being involved in numerous biological processes, including tumorigenesis (34). RhoA, as the most extensively investigated member of the Rho protein family, acts as a molecular switch in cells, regulating signal transduction from cell surface receptors to intracellular target molecules, and is involved in a number of biological processes, including cell morphology, motility and tumor progression (34). Without destroying the cells, the present study demonstrated a redistribution of nucleolar RhoA between the nucleoli and the nucleoplasm with a speckled staining pattern following the treatment of HEp-2 cells with actinomycin D. Furthermore, immunoblotting revealed a single band exclusively at $21 \mathrm{kDa}$, indicating that an actinomycin D-induced decrease in nucleolar RhoA was not accompanied by any alteration in RhoA protein content, biochemical modification or cleavage of RhoA. These results are concordant with previous findings regarding the nucelolar protein fibrillarin following treatment with actinomycin D. The present results demonstrating that the nucleolar protein B23 translocated to the nucleoplasm, along with redistribution of nucleolar fibrillarin to the nucleoplasm following treatment with actinomycin $\mathrm{D}$, are also consistent with previous observations made in various types of cells $(24,28)$. As actinomycin D may displace RNA polymerases by binding DNA and subsequently inhibiting RNA synthesis (35), the present results may reflect the redistribution of RhoA between the nucleoli and nucleoplasm when RNA synthesis is inhibited.

At low concentrations (0.04-0.05 mg/l), actinomycin D selectively inhibits transcription that is mediated by RNA polymerase I in the nucleolus, but not transcription mediated by RNA polymerase II and III (36). The reaccumulation of RhoA occurred between the nucleoplasm and the nucleoli following removal of the lower concentration $(0.05 \mathrm{mg} / \mathrm{l})$ of actinomycin $\mathrm{D}$, which extended the aforementioned results due to the observation that the effect on the subcellular redistribution of RhoA induced by low concentrations of actinomycin D was reversible. However, due to the action of actinomycin D on RNA polymerases II and III at increased concentrations, even subsequent to an extended period of drug removal, the displacement of RhoA was irreversible upon treatment of cells with increased concentrations of actinomycin $\mathrm{D}$, which led to severe inhibition of RNA synthesis. The DFC and the edge of the FC are the nucleolar regions where transcription takes place (7). Although the exact location of RhoA in respect to the nucleolar component remains to be elucidated, considering the similar nucleolar localization of RhoA and transcription sites, the observation of actinomycin D-induced redistribution of nucleolar RhoA to the nucleoplasm revealed direct evidence that the residency of RhoA in the nucleolus was dependent on active transcription.

In line with the present results, the $\beta$ II-subunit of tubulin, a major subunit of microtubules that was previously considered to exist solely in the cytoplasm of cells, has also been identified to localize to the nuclei, with a strong aggregation in the nucleoli of numerous types of carcinoma cells $(37,38)$. Following treatment with the drug taxol, which inhibits mitosis targeted at the microtubules of dividing cells, $\beta$ II-tubulin underwent a rearrangement in the nuclei and demonstrated the formation of micronuclei (37). Increasing evidence emerged that these cellular proteins may be dynamic, crossing through the cytoplasm, nucleoplasm and nucleolus to perform their roles, and a number of these proteins may remain to be elucidated $(16,18,38)$.

In the current era of translational medicine, the newly recognized roles of the nucleolus raise the possibility that cancer drug discovery and emerging chemotherapy may identify a foundation in the nucleolus or nucleolar proteins to a greater degree than had been anticipated (35). Knowledge of the location, function and duration of the residency of proteins in the nucleolus is vital in identifying additional drug targets in the nucleolus.

In conclusion, the results of the present study provided in situ evidence that actinomycin $\mathrm{D}$ induced a redistribution of nucleolar RhoA to the nucleoplasm in human carcinoma HEp-2 cells. In addition, the results indicated that the residency of RhoA in the cell nucleolus is associated with active RNA synthesis. To the best of our knowledge, these results provide the first information regarding nucleolar RhoA in association with nucleolar function. Additional investigations on the nuclear or nucleolar functioning of RhoA are underway and may provide novel considerations for cancer therapy.

\section{Acknowledgements}

The present study was supported by the National Natural Science Foundation of China (Beijing, China; grant no. 81372404), the Specialized Research Fund for Senior Personnel Program of Jiangsu University (Zhenjiang, China; grant no. 11JDG129) and the Postdoctoral Foundations of China (Beijing, China; grant no. 2012M521018) and Jiangsu province (Nanjing, China; grant no. $1201025 B)$ granted to Dr Yueying Li.

\section{References}

1. Misteli T: Protein dynamics: Implications for nuclear architecture and gene expression. Science 291: 843-847, 2001.

2. Scheer U, Thiry M and Goessens G: Structure, function and assembly of the nucleolus. Trends Cell Biol 3: 236-241, 1993.

3. Bártová E, Horáková AH, Uhlírová R, Raska I, Galiová G, Orlova D and Kozubek S: Structure and epigenetics of nucleoli in comparison with non-nucleolar compartments. J Histochem Cytochem 58: 391-403, 2010.

4. Hernandez-Verdun D: The nucleolus today. J Cell Sci 99:465-471, 1991.

5. Spector DL: Macromolecular domains within the cell nucleus. Annu Rev Biochem 9: 265-315, 1993.

6. Shaw PJ and Jordan EG: The nucleolus. Annu Rev Cell Dev Biol 11: 93-121, 1995.

7. Scheer U and Hock R: Structure and function of the nucleolus. Curr Opin Cell Biol 11: 385-390, 1999. 
8. Muro E, Gébrane-Younís J, Jobart-Malfait A, Louvet E, Roussel P and Hernandez-Verdun D: The traffic of proteins between nucleolar organizer regions and prenucleolar bodies governs the assembly of the nucleolus at exit of mitosis. Nucleus 1: 201-211, 2010.

9. Pederson T: The plurifunctional nucleolus. Nuclei Acid Res 26: 3871-3876, 1998.

10. Pederson T: The nucleolus. Cold Spring Harb Perspect Biol 3: a000638, 2011.

11. Mackay DJ and Hall A: Rho GTPases. J Biol Chem 273 20685-20688, 1998.

12. Sotiropoulos A, Gineitis D, Copeland J and Treisman R Signal-regulated activation of serum response factor is mediated by changes in actin dynamics. Cell 98: 159-169, 1999.

13. Kamai T, Yamanishi T, Shirataki H, Takagi K, Asami H, Ito Y and Yoshida K: Overexpression of RhoA, Rac1, and Cdc42 GTPases is associated with progression in testicular cancer. Clin Cancer Res 10: 4799-4805, 2004.

14. Dubash AD, Guilluy C, Srougi MC, Boulter E, Burridge K and García-Mata R: The small GTPase RhoA localizes to the nucleus and is activated by Net1 and DNA damage signals. PloS One 6: e17380, 2011

15. Guilluy C, Dubash AD and García-Mata R: Analysis of RhoA and Rho GEF activity in whole-cells and the cell nucleus. Nat Protoc 6: 2050-2060, 2011

16. Li YY, Chen YC and Xu J: Factors influencing RhoA protein distribution in the nucleus. Mol Med Report 4: 1115-1119, 2011.

17. Li YY, Chen YC, Tao Y, Xu J and Chen M: RhoA protein is generally distributed in the nuclei of cancer cells. Oncol Rep 24: 1005-1009, 2010.

18. Xu J, Li Y, Yang X, Chen Y and Chen M: Nuclear translocation of Small G protein RhoA via active transportation in gastric cancer cells. Oncol Rep 30: 1878-1882, 2013.

19. Perry RP and Kelly DE: Inhibition of RNA sythesis by actinomycin D: Characteristic dose-response of different RNA species. J Cell Physiol 76: 127-139, 1970.

20. Chen FM: Binding specificities of actinomycin D to self-complementary tetranucleotide sequences -XGCY-. Biochemistry 27: 6393-6397, 1988

21. Biggiogera M, Fakan S, Kaufmann SH, Black A, Shaper JH and Busch H: Simultaneous immunoelectron microscopic visualization of protein B23 and C23 distribution in the HeLa cell nucleolus. J Histochem Cytochem 37: 1371-1374, 1989.

22. Sweet P, Chan PK and Slater LM: Cyclosporin A and verapamil enhancement of daunorubicin-produced nucleolar protein B23 translocation in daunorubicin-resistant and -sensitive human and murine tumor cells. Cancer Res 49: 677-680, 1989.

23. Yung BY, Bor AM and Chan PK: Short exposure to actinomycin D induced 'reversible' translocation of protein B23 as well as 'reversible' inhibition of cell growth and RNA synthesis in HeLa cells. Cancer Res 50: 5987-5991, 1990.
24. Yao Z, Duan S, Hou D, Wang W, Wang G, Liu Y, Wen L and Wu M: B23 acts as a nucleolar stress sensor and promotes cell survival through its dynamic interaction with hnRNPU and hnRNPA1. Oncogene 29: 1821-1834, 2010.

25. Benavente R, Reimer G, Rose KM, Hügle-Dörr B and Scheer U: Nucleolar changes after microinjection of antibodies to RNA polymerase I into the nucleus of mammalian cells. Chromosoma 97: 115-123, 1988.

26. Puvion-Dutilleul F, Mazan S, Nicoloso M, Pichard E, Bachellerie JP and Puvion E: Alteration of nucleolarultrastructure and ribosome biogenesis by actinomycin D. Implications for U3 snRNP function. Eur J Cell Biol 58: 149-162, 1992.

27. Rivera-León R and Gerbi SA: Delocalization of some small nucleolar RNPs after actinomycin D treatment to deplete early pre-rRNAs. Chromosoma 105: 506-514, 1997.

28. Chen M and Jiang P: Altered subcellular distribution of nucleolar protein fibrillarin by actinomycin D in HEp-2 cells. Acta Pharmacol Sin 25: 902-906, 2004

29. Boisvert FM, van Koningsbruggen S, Navascués J and Lamond AI: The multifunctional nucleolus. Nat Rev Mol Cell Biol 8: 574-585, 2007.

30. Montanaro L, Treré D and Derenzini M: Nucleolus, ribosomes, and cancer. Am J Pathol 173: 301-310, 2008.

31. Hein N, Hannan KM, George AJ, Sanij E and Hannan RD: The nucleolus: An emerging target for cancer therapy. Trends Mol Med 19: 643-654, 2013.

32. Pianese G: Beitrag zur Histologie und Aetiologie der Carcinoma Histologische und experimentelle Untersuchungen. Beitr Pathol Anat Allg Pathol 142: 1-193, 1896.

33. Quin JE, Devlin JR, Cameron D, Hannan KM, Pearson RB and Hannan RD: Targeting the nucleolus for cancer intervention. Biochim Biophys Acta 1842: 802-816, 2014.

34. Orgaz JL, Herraiz C and Sanz-Moreno V: Rho GTPases modulate malignant transformation of tumor cells. Small GTPases 5: e29019, 2014

35. Pickard AJ and Bierbach U: The cell's nucleolus: An emerging target for chemotherapeutic intervention. Chem Med Chem 8: 1441-1449, 2013.

36. Ochs RL: Methods used to study structure and function of the nucleolus. Methods Cell Biol 53: 303-321, 1998.

37. Xu K and Ludueña RF: Characterization of nuclear betaII-tubulin in tumor cells: A possible novel target for taxol. Cell Motil Cytoskeleton 53: 39-52, 2002

38. Howard $\mathbf{J}$ and Hyman AA: Dynamics and mechanics of the microtubule plus end. Nature 422: 753-758, 2003. 